\title{
The Hermite-Hadamard's inequality for some convex functions via fractional integrals and related results
}

\author{
E. SET, M. Z. SARIKAYA, M. E. ÖZDEMIR AND H. YILDIRIM
}

\begin{abstract}
In this paper, we establish Hermite-Hadamard type inequalities for $s$-convex functions in the second sense and $m$-convex functions via fractional integrals. The analysis used in the proofs is fairly elementary.
\end{abstract}

Mathematics Subject Classification 2000: 26D07, 26D10, 26D15, 47A63

Additional Key Words and Phrases: $s$-convex functions, Hermite-Hadamard type inequalities, Riemann-Liouville fractional integral.

\section{INTRODUCTION}

Let real function $f$ be defined on some nonempty interval $I$ of real line $\mathrm{R}$. The function $f$ is said to be convex on $I$ if inequality

$$
f(\lambda x+(1-\lambda) y) \leq \lambda f(x)+(1-\lambda) f(y)
$$

holds for all $x, y \in I$ and $\lambda \in[0,1]$.

In [10], Hudzik and Maligranda considered, among others, the class of functions which are $s$-convex in the second sense. This class of functions is defined as the following:

DEFINITION 1. A function $f:[0, \infty) \rightarrow \mathrm{R}$ is said to be $s$-convex in the second sense if

$$
f(\lambda x+(1-\lambda) y) \leq \lambda^{s} f(x)+(1-\lambda)^{s} f(y)
$$

for all $x, y \in[0, \infty), \lambda \in[0,1]$ and for some fixed $s \in(0,1]$. This class of $s-$ convex functions is usually denoted by $K_{s}^{2}$. 
It can be easily seen that for $s=1, s$-convexity reduces to ordinary convexity of functions defined on $[0, \infty)$.

In [12], G. Toader considered the class of $m$-convex functions: another intermediate between the usual convexity and starshaped convexity.

DEFINITION 2. The function $f:[0, b] \rightarrow \mathrm{R}, \quad b>0$, is said to be $m$-convex, where $m \in[0,1]$, if we have

$$
f(t x+m(1-t) y) \leq t f(x)+m(1-t) f(y)
$$

for all $x, y \in[0, b]$ and $t \in[0,1]$. We say that $f$ is $m$-concave if $(-f)$ is $m$-convex.

Obviously, for $m=1$ Definition D.1 recaptures the concept of standard convex functions on $[a, b]$, and for $m=0$ the concept starshaped functions.

One of the most famous inequalities for convex functions is HermiteHadamard inequality. This double inequality is stated as follows (see for example [5] and [14]): Let $f$ be a convex function on some nonempty interval $[a, b]$ of real line $\mathrm{R}$, where $a \neq b$. Then

$$
f\left(\frac{a+b}{2}\right) \leq \frac{1}{b-a} \int_{a}^{b} f(x) d x \leq \frac{f(a)+f(b)}{2} .
$$

Both inequalities hold in the reversed direction if $f$ is concave. We note that Hermite-Hadamard inequality may be regarded as a refinement of the concept of convexity and it follows easily from Jensen's inequality. Hermite-Hadamard inequality for convex functions has received renewed attention in recent years and a remarkable variety of refinements and generalizations have been found (see, for example, [1]-[16]).

In [8], Hermite-Hadamard inequality for $s$-convex functions in the second sense is proved by Dragomir et. al. 
THEOREM 1. Suppose that $f:[0, \infty) \rightarrow[0, \infty)$ is an $s$-convex function in the second sense, where $s \in(0,1)$, and let $a, b \in[0, \infty), \quad a<b$. If $f \in L^{1}([a, b])$, then the following inequalities hold:

$$
2^{s-1} f\left(\frac{a+b}{2}\right) \leq \frac{1}{b-a} \int_{a}^{b} f(x) d x \leq \frac{f(a)+f(b)}{s+1} .
$$

The constant $k=\frac{1}{s+1}$ is the best possible in the second inequality in (1.2).

In [11], Kirmaci et. al. established a new Hermite-Hadamard type inequality which holds for $s$-convex functions in the second sense. It is given in the next theorem.

THEOREM 2. Let $f: I \rightarrow \mathrm{R}, \quad I \subset[0, \infty)$, be a differentiable function on $I^{\circ} \quad$ such that $f^{\prime} \in L_{1}([a, b])$, where $a, b \in I, \quad a<b$. If $\left|f^{\prime}\right|^{q}$ is $s$-convex on $[a, b]$ for some fixed $s \in(0,1)$ and $q \geq 1$, then:

$$
\begin{aligned}
& \left|\frac{f(a)+f(b)}{2}-\frac{1}{b-a} \int_{a}^{b} f(x) d x\right| \\
& \leq \frac{b-a}{2}\left(\frac{1}{2}\right)^{\frac{q-1}{q}}\left[\frac{s+\left(\frac{1}{2}\right)^{s}}{(s+1)(s+2)}\right]^{\frac{1}{q}}\left[\left|f^{\prime}(a)\right|^{q}+\left|f^{\prime}(b)\right|^{q}\right]^{\frac{1}{q}} .
\end{aligned}
$$

We give some necessary definitions and mathematical preliminaries of fractional calculus theory which are used throughout this paper.

DEFINITION 3. Let $f \in L_{1}[a, b]$. The Riemann-Liouville integrals $J_{a+}^{\alpha} f$ and $J_{b-}^{\alpha} f$ of order $\alpha>0$ with $a \geq 0$ are defined by

$$
J_{a+}^{\alpha} f(x)=\frac{1}{\Gamma(\alpha)} \int_{a}^{x}(x-t)^{\alpha-1} f(t) d t, \quad x>a
$$

and

$$
J_{b-}^{\alpha} f(x)=\frac{1}{\Gamma(\alpha)} \int_{x}^{b}(t-x)^{\alpha-1} f(t) d t, \quad x<b
$$


respectively where $\Gamma(\alpha)=\int_{0}^{\infty} e^{-t} u^{\alpha-1} d u$. Here is $J_{a_{+}}^{0} f(x)=J_{b_{-}}^{0} f(x)=f(x)$.

In the case of $\alpha=1$, the fractional integral reduces to the classical integral. Properties concerning this operator can be found ([24],[25] and [26]).

For some recent results connected with fractional integral inequalities see ([18]-[29])

In [28] Sarkaya et. al. proved a variant of the identity established by Dragomir et. al. in [6] for fractional integrals which is stated in the following lemma.

LEMMA 1. Let $f:[a, b] \rightarrow \mathrm{R}$, be a differentiable mapping on $(a, b)$ with $a<b$. If $f^{\prime} \in L[a, b]$, then the following equality for fractional integrals holds:

$$
\begin{aligned}
& \frac{f(a)+f(b)}{2}-\frac{\Gamma(\alpha+1)}{2(b-a)^{\alpha}}\left[J_{a+}^{\alpha} f(b)+J_{b-}^{\alpha} f(a)\right] \\
= & \frac{b-a}{2} \int_{0}^{1}\left[(1-t)^{\alpha}-t^{\alpha}\right] f^{\prime}(t a+(1-t) b) d t .
\end{aligned}
$$

The aim of this paper is to establish Hermite-Hadamard inequality and Hermite-Hadamard type inequalities for $s$-convex functions in the second sense and $m$-conex functions via Riemann-Liouville fractional integral. 


\section{HERMITE-HADAMARD TYPE INEQUALITIES FOR SOME CONVEX FUNCTIONS VIA FRACTIONAL INTEGRALS}

\subsection{For $s$-convex functions}

Hermite-Hadamard inequality can be represented for $s$-convex functions in fractional integral forms as follows:

THEOREM 3. Let $f:[a, b] \rightarrow \mathrm{R}$ be a positive function with $0 \leq a<b$ and $f \in L_{1}[a, b]$. If $f$ is a $s$-convex mapping in the second sense on $[a, b]$, then the following inequalities for fractional integrals with $\alpha>0$ and $s \in(0,1)$ hold:

$$
\begin{aligned}
2^{s-1} f\left(\frac{a+b}{2}\right) & \leq \frac{\Gamma(\alpha+1)}{(b-a)^{\alpha}}\left[\frac{J_{a+}^{\alpha} f(b)+J_{b-}^{\alpha} f(a)}{2}\right] \\
& \leq\left[\frac{1}{(\alpha+s)}+\beta(\alpha, s+1)\right] \frac{f(a)+f(b)}{2}
\end{aligned}
$$

where $\beta$ is Euler Beta function.

PROOF. Since $f$ is a $s$-convex mapping in the second sense on $[a, b]$, we have for $x, y \in[a, b]$ with $\lambda=\frac{1}{2}$

$$
f\left(\frac{x+y}{2}\right) \leq \frac{f(x)+f(y)}{2^{s}} .
$$

Now, let $x=t a+(1-t) b$ and $y=(1-t) a+t b$ with $t \in[0,1]$ Then, we get by (2.2) that:

$$
2^{s} f\left(\frac{a+b}{2}\right) \leq f(t a+(1-t) b)+f((1-t) a+t b)
$$

for all $t \in[0,1]$

Multiplying both sides of (2.3) by $t^{\alpha-1}$, then integrating the resulting inequality with respest to $t$ over $[0,1]$, we obtain 


$$
\begin{aligned}
& \frac{2^{s}}{\alpha} f\left(\frac{a+b}{2}\right) \\
\leq & \int_{0}^{1} t^{\alpha-1} f(t a+(1-t) b) d t+\int_{0}^{1} t^{\alpha-1} f((1-t) a+t b) d t \\
= & \frac{1}{(b-a)^{\alpha}} \int_{a}^{b}(b-u)^{\alpha-1} f(u) d u-\frac{1}{(a-b)^{\alpha}} \int_{a}^{b}(a-v)^{\alpha-1} f(v) d v \\
= & \frac{\Gamma(\alpha)}{(b-a)^{\alpha}}\left[J_{a+}^{\alpha} f(b)+J_{b-}^{\alpha} f(a)\right]
\end{aligned}
$$

i.e.

$$
2^{s-1} f\left(\frac{a+b}{2}\right) \leq \frac{\Gamma(\alpha+1)}{(b-a)^{\alpha}}\left[\frac{J_{a+}^{\alpha} f(b)+J_{b-}^{\alpha} f(a)}{2}\right]
$$

and the first inequality is proved.

For the proof of the second inequality in (2.1) we first note that if $f$ is a $s$-convex mapping in the second sense, then, for $t \in[0,1]$, it yields

$$
f(t a+(1-t) b) \leq t^{s} f(a)+(1-t)^{s} f(b)
$$

and

$$
f((1-t) a+t b) \leq(1-t)^{s} f(a)+t^{s} f(b) .
$$

By adding these inequalities we have

$$
f(t a+(1-t) b)+f((1-t) a+t b) \leq\left\lfloor t^{s}+(1-t)^{s}\right\rfloor(f(a)+f(b)) .
$$

Thus, multiplying both sides of (24) by $t^{\alpha-1}$ and integrating the resulting inequality with respest to $t$ over $[0,1]$, we obtain

$$
\begin{aligned}
& \int_{0}^{1} t^{\alpha-1} f(t a+(1-t) b) d t+\int_{0}^{1} t^{\alpha-1} f((1-t) a+t b) d t \\
\leq & {[f(a)+f(b)] \int_{0}^{1} t^{\alpha-1}\left[t^{s}+(1-t)^{s}\right] d t }
\end{aligned}
$$


i.e.

$$
\frac{\Gamma(\alpha)}{(b-a)^{\alpha}}\left[J_{a+}^{\alpha} f(b)+J_{b-}^{\alpha} f(a)\right] \leq[1+(\alpha+s) \beta(\alpha, s+1)] \frac{f(a)+f(b)}{(\alpha+s)}
$$

where the proof is completed.

REMARK 1. If we choose $\alpha=1$ in Theorem 3, then the inequalities (2.1) become the inequalities (1.2) of Theorem 1.

Using Lemma 1, we can obtain the following fractional integral inequality for $s$-convex in the second sense:

THEOREM 4. Let $f:[a, b] \subset[0, \infty) \rightarrow \mathrm{R}$ be a differentiable mapping on $(a, b)$ with $a<b$ such that $f^{\prime} \in L[a, b]$. If $\left|f^{\prime}\right|^{q}$ is $s$-convex in the second sense on $[a, b]$ for some fixed $s \in(0,1)$ and $q \geq 1$, then the following inequality for fractional integrals holds:

$$
\begin{aligned}
& \mid \frac{f(a)+f(b)}{2}-\frac{\Gamma(\alpha+1)}{2(b-a)^{\alpha}}\left[J_{a+}^{\alpha} f(b)+J_{b-}^{\alpha} f(a)\right] \\
& \leq \frac{b-a}{2}\left[\frac{2}{\alpha+1}\left(1-\frac{1}{2^{\alpha}}\right)\right]^{\frac{q-1}{q}}\left\{\beta\left(\frac{1}{2} ; s+1, \alpha+1\right)-\beta\left(\frac{1}{2} ; \alpha+1, s+1\right)\right. \\
& \left.+\frac{2^{\alpha+s}-1}{(\alpha+s+1) 2^{\alpha+s}}\right\}\left(\left|f^{\prime}(a)\right|^{q}+\left|f^{\prime}(b)\right|^{q}\right)^{\frac{1}{q}} .
\end{aligned}
$$

PROOF. Suppose that $q=1$. From Lemma 1 and using the properties of modulus, we have

$$
\begin{aligned}
& \mid \frac{f(a)+f(b)}{2}-\frac{\Gamma(\alpha+1)}{2(b-a)^{\alpha}}\left[J_{a+}^{\alpha} f(b)+J_{b-}^{\alpha} f(a)\right] \\
& \leq \frac{b-a}{2} \int_{0}^{1}\left|(1-t)^{\alpha}-t^{\alpha}\right|\left|f^{\prime}(t a+(1-t) b)\right| d t .
\end{aligned}
$$


Since $\left|f^{\prime}\right|$ is $s$-convex on $[a, b]$, we have

$$
\begin{aligned}
& \mid \frac{f(a)+f(b)}{2}-\frac{\Gamma(\alpha+1)}{2(b-a)^{\alpha}}\left[J_{a+}^{\alpha} f(b)+J_{b-}^{\alpha} f(a)\right] \\
& \leq \frac{b-a}{2} \int_{0}^{1}\left|(1-t)^{\alpha}-t^{\alpha}\right|\left[t^{s}\left|f^{\prime}(a)\right|+(1-t)^{s}\left|f^{\prime}(b)\right|\right] d t \\
& =\frac{b-a}{2}\left\{\int_{0}^{\frac{1}{2}}\left[(1-t)^{\alpha}-t^{\alpha}\right]\left[t^{s}\left|f^{\prime}(a)\right|+(1-t)^{s}\left|f^{\prime}(b)\right|\right] d t\right. \\
& \left.+\int_{\frac{1}{2}}^{1}\left[t^{\alpha}-(1-t)^{\alpha}\right]\left[t^{s}\left|f^{\prime}(a)\right|+(1-t)^{s}\left|f^{\prime}(b)\right|\right] d t\right\} \\
& +\frac{b-a}{2}\left\{\mid f^{\prime}(a)\right)\left|\int_{0}^{\frac{1}{2}} t^{s}(1-t)^{\alpha} d t-\right| f^{\prime}(a) \mid \int_{0}^{\frac{1}{2}} t^{s+\alpha} d t \\
& \left.\left.\left.\left.\left.+\mid f^{\prime}(a)\right)\left|\int_{\frac{1}{2}}^{1} t^{\alpha+s} d t-\right| f^{\prime}(a)\right) \mid \int_{\frac{1}{2}}^{1} t^{s}(1-t)^{\alpha} d t\right)\left|\int_{0}^{\frac{1}{2}}(1-t)^{s+\alpha} d t-\right| f^{\prime}(b)\right) \mid \int_{0}^{\frac{1}{2}}(1-t)^{s} t^{\alpha} d t\right] \\
& +
\end{aligned}
$$


Since

$$
\begin{aligned}
\int_{0}^{\frac{1}{2}} t^{s}(1-t)^{\alpha} d t & =\int_{\frac{1}{2}}^{1}(1-t)^{s} t^{\alpha} d t=\beta\left(\frac{1}{2} ; s+1, \alpha+1\right), \\
\int_{0}^{\frac{1}{2}}(1-t)^{s} t^{\alpha} d t & =\int_{\frac{1}{2}}^{1} t^{s}(1-t)^{\alpha} d t=\beta\left(\frac{1}{2} ; \alpha+1, s+1\right), \\
\int_{0}^{\frac{1}{2}} t^{s+\alpha} d t & =\int_{\frac{1}{2}}^{1}(1-t)^{s+\alpha} d t=\frac{1}{2^{s+\alpha+1}(s+\alpha+1)}
\end{aligned}
$$

and

$$
\int_{0}^{\frac{1}{2}}(1-t)^{s+\alpha} d t=\int_{\frac{1}{2}}^{1} t^{s+\alpha} d t=\frac{1}{(s+\alpha+1)}-\frac{1}{2^{s+\alpha+1}(s+\alpha+1)}
$$

We obtain

$$
\begin{aligned}
& \mid \frac{f(a)+f(b)}{2}-\frac{\Gamma(\alpha+1)}{2(b-a)^{\alpha}}\left[J_{a+}^{\alpha} f(b)+J_{b-}^{\alpha} f(a) \mid\right. \\
& \leq \frac{b-a}{2}\left[\left|f^{\prime}(a)\right|+\left|f^{\prime}(b)\right|\right] \\
& \quad \times\left\{\beta\left(\frac{1}{2} ; s+1, \alpha+1\right)-\beta\left(\frac{1}{2} ; \alpha+1, s+1\right)+\frac{2^{\alpha+s}-1}{(\alpha+s+1) 2^{\alpha+s}}\right\}
\end{aligned}
$$

which completes the proof for this case. Suppose now that $q>1$. Since $\left|f^{\prime}\right|^{q}$ is $s$-convex on $[a, b]$, we know that for every $t \in[0,1]$

$$
\left|f^{\prime}(t a+(1-t) b)\right|^{q} \leq t^{s}\left|f^{\prime}(a)\right|^{q}+(1-t)^{s}\left|f^{\prime}(b)\right|^{q},
$$

so using well know Hölder's inequality (see for example [17]) for $\frac{1}{p}+\frac{1}{q}=1,(q>1)$ and (2.8) in (2.6), we have successively 


$$
\begin{aligned}
& \mid \frac{f(a)+f(b)}{2}-\frac{\Gamma(\alpha+1)}{2(b-a)^{\alpha}}\left[J_{a+}^{\alpha} f(b)+J_{b-}^{\alpha} f(a) \mid\right. \\
\leq & \frac{b-a}{2} \int_{0}^{1}\left|(1-t)^{\alpha}-t^{\alpha}\right|\left|f^{\prime}(t a+(1-t) b)\right| d t \\
= & \frac{b-a}{2} \int_{0}^{1}\left|(1-t)^{\alpha}-t^{\alpha}\right|^{1-\frac{1}{q}}\left|(1-t)^{\alpha}-t^{\alpha}\right|^{\frac{1}{q}}\left|f^{\prime}(t a+(1-t) b)\right| d t \\
\leq & \frac{b-a}{2}\left(\int_{0}^{1}\left|(1-t)^{\alpha}-t^{\alpha}\right| d t\right)^{\frac{q-1}{q}}\left(\int_{0}^{1}\left|(1-t)^{\alpha}-t^{\alpha}\right|\left|f^{\prime}(t a+(1-t) b)\right|^{q} d t\right)^{\frac{1}{q}} \\
\leq & \frac{b-a}{2}\left[\frac{2}{\alpha+1}\left(1-\frac{1}{2^{\alpha}}\right)\right]^{\frac{q-1}{q}}\left(\left|f^{\prime}(a)\right|^{q}+\left|f^{\prime}(b)\right|^{q}\right)^{\frac{1}{q}} \\
& \left.\times\left\{\frac{\beta}{2} ; s+1, \alpha+1\right)-\beta\left(\frac{1}{2} ; s+1, \alpha+1\right)+\frac{2^{\alpha+s}-1}{(\alpha+s+1) 2^{\alpha+s}}\right\}
\end{aligned}
$$

where we use the fact that

$$
\begin{aligned}
\int_{0}^{1}\left|(1-t)^{\alpha}-t^{\alpha}\right| d t & =\int_{0}^{\frac{1}{2}}\left[(1-t)^{\alpha}-t^{\alpha}\right] d t+\int_{\frac{1}{2}}^{1}\left[t^{\alpha}-(1-t)^{\alpha}\right] d t \\
& =\frac{2}{\alpha+1}\left(1-\frac{1}{2^{\alpha}}\right)
\end{aligned}
$$

which completes the proof.

REMARK 2. If we take $\alpha=1$ in Theorem 4, then the inequality (2.5) becomes the inequality (1.3) of Theorem 2. 


\subsection{For $m$-convex functions}

We start with the following theorem:

THEOREM 5. Let $f:[0, \infty] \rightarrow \mathrm{R}$ be a positive function with $0 \leq a<b$ and $f \in L_{1}[a, b]$. If $f$ is $m$-convex mapping on $[a, b]$, then the following inequalities for fractional integral with $\alpha>0$ and $m \in(0,1]$ hold:

$$
\begin{aligned}
\frac{2}{\Gamma(\alpha+1)} f\left(\frac{m(a+b)}{2}\right) & \leq \frac{1}{(m b-m a)^{\alpha}} J_{(m a)^{+}}^{\alpha} f(m b)+\frac{m}{(b-a)^{\alpha}} J_{b^{-}}^{\alpha} f(a) \\
& \leq \frac{f(m a)+m^{2} f\left(\frac{b}{m}\right)}{(\alpha+1)}+m \frac{f(a)+f(b)}{\alpha(\alpha+1)}
\end{aligned}
$$

PROOF. Since $f$ is $m$-convex functions, we have

$$
f(t x+m(1-t) y) \leq t f(x)+m(1-t) f(y)
$$

and if we choose $t=\frac{1}{2}$, we get

$$
f\left(\frac{1}{2}(x+m y)\right) \leq \frac{f(x)+m f(y)}{2} .
$$

Now, let $x=m t a+m(1-t) b$ and $y=(1-t) a+t b$ with $t \in[0,1]$. Then we get

$$
f\left(\frac{1}{2} m(a+b)\right) \leq \frac{f(m t a+m(1-t) b)+m f((1-t) a+t b)}{2} .
$$

Multiplying both sides of and (2.10) by $t^{\alpha-1}$, then integrating the resulting inequlities with respect to $t$ over $[0,1]$, we obtain 


$$
\begin{aligned}
& \frac{1}{\alpha} f\left(\frac{1}{2} m(a+b)\right) \\
\leq & \frac{1}{2} \int_{0}^{1} t^{\alpha-1} f(m t a+m(1-t) b) d t+\frac{m}{2} \int_{0}^{1} t^{\alpha-1} f((1-t) a+t b) d t \\
= & \frac{1}{2} \int_{m b}^{m a}\left(\frac{u-m b}{m a-m b}\right)^{\alpha-1} f(u) \frac{d u}{m(a-b)}+\frac{m}{2} \int_{a}^{b}\left(\frac{v-a}{b-a}\right)^{\alpha-1} f(v) \frac{d v}{b-a} \\
= & \frac{1}{2(m b-m a)^{\alpha}} \int_{m a}^{m b}(m b-u)^{\alpha-1} f(u) d u+\frac{m}{2} \frac{\Gamma(\alpha)}{(b-a)^{\alpha}} J_{b^{-}}^{\alpha} f(a)
\end{aligned}
$$

which the first inequality is proved.

By the $m$-convexity of $f$, we also have

$$
\begin{aligned}
& \frac{1}{2}[f(m t a+m(1-t) b)+m f((1-t) a+t b)] \\
\leq & \frac{1}{2}\left[m t f(a)+m(1-t) f(b)+m(1-t) f(a)+m^{2} f\left(\frac{b}{m}\right)\right]
\end{aligned}
$$

for all $t \in[0,1]$. Multiplying both sides of above inequality by $t^{\alpha-1}$ and integrating over $t \in[0,1]$, we get

$$
\begin{aligned}
& \frac{1}{(m b-m a)^{\alpha}} \int_{m a}^{m b}(m b-u)^{\alpha-1} f(u) d u+\frac{m}{(b-a)^{\alpha}} \int_{a}^{b}(v-a)^{\alpha-1} f(v) d v \\
\leq & \frac{f(m a)+m^{2} f\left(\frac{b}{m}\right)}{(\alpha+1)}+m \frac{f(a)+f(b)}{\alpha(\alpha+1)}
\end{aligned}
$$

which this gives the second part of (2.9).

COROLLARY 1. Under the conditions in Theorem 5 with $\alpha=1$, then the following inequality hold:

$$
\begin{aligned}
f\left(\frac{m(a+b)}{2}\right) & \leq \frac{1}{(b-a)} \int_{a}^{b} \frac{f(m x)+m f(x)}{2} d x \\
& \leq \frac{1}{2}\left[\frac{f(m a)+m^{2} f\left(\frac{b}{m}\right)}{2}+m \frac{f(a)+f(b)}{2}\right] .
\end{aligned}
$$


REMARK 3. If we take $m=1$ in Corollary $k$, then the inequalities (2.11) become the inequalities (1.1).

THEOREM 6. Let $f:[0, \infty] \rightarrow \mathrm{R}$, be $m$-convex functions with $m \in(0,1]$, $0 \leq a<b$ and $f \in L_{1}[a, b] . \quad F(x, y)_{(t)}:[0,1] \rightarrow \mathrm{R}$ are defined as the following:

$$
F(x, y)_{(t)}=\frac{1}{2}[f(t x+m(1-t) y)+f((1-t) x+m t y)] .
$$

Then, we have

$$
\frac{1}{(b-a)^{\alpha}} \int_{a}^{b}(b-u)^{\alpha-1} F\left(u, \frac{a+b}{2}\right)_{\left(\frac{b-u}{b-a}\right)} d u \leq \frac{\Gamma(\alpha)}{2(b-a)^{\alpha}} J_{a^{+}}^{\alpha} f(b)+\frac{m}{2 \alpha} f\left(\frac{a+b}{2}\right)
$$

for all $t \in[0,1]$.

PROOF. Since $f$ and $g$ are $m$-convex functions, we have

$$
\begin{aligned}
F(x, y)_{(t)} & \leq \frac{1}{2}[t f(x)+m(1-t) f(y)+(1-t) f(x)+m t f(y)] \\
& =\frac{1}{2}[f(x)+m f(y)]
\end{aligned}
$$

and so,

$$
F\left(x, \frac{a+b}{2}\right)_{(t)} \leq \frac{1}{2}\left[f(x)+m f\left(\frac{a+b}{2}\right)\right] .
$$

If we choose $x=t a+(1-t) b$, we have

$$
F\left(t a+(1-t) b, \frac{a+b}{2}\right)_{(t)} \leq \frac{1}{2}\left[f(t a+(1-t) b)+m f\left(\frac{a+b}{2}\right)\right]
$$

Thus multiplying both sides of (2.12) by $t^{\alpha-1}$, then integrating the resulting inequality with respect to $t$ over $[0,1]$, we obtain

$$
\begin{aligned}
& \int_{0}^{1} t^{\alpha-1} F\left(t a+(1-t) b, \frac{a+b}{2}\right){ }_{(t)} d t \\
& \leq \frac{1}{2}\left[\int_{0}^{1} t^{\alpha-1} f(t a+(1-t) b) d t+\int_{0}^{1} t^{\alpha-1} m f\left(\frac{a+b}{2}\right) d t\right] .
\end{aligned}
$$


Thus, if we use the change of the variable $u=t a+(1-t) b, t \in[0,1]$, then have the conclusion.

\section{REFERENCES}

[1] Alomari, M., Darus, M., On the Hadamard's inequality for log-convex functions on the coordinates, Journal of Inequalities and Applications, vol. 2009, Article ID 283147, 13 pages, 2009.

[2] Azpeitia, A.G., Convex functions and the Hadamard inequality, Rev. Colombiana Math., 28 (1994), 7-12.

[3] Bakula, M.K., Özdemir, M.E., Pečarić, J., Hadamard tpye inequalities for $m$-convex and $(\alpha, m)$-convex functions, J. Ineq. Pure and Appl. Math., 9(4) (2008), Art. 96.

[4] Bakula, M. K., Pečarić, J., Note on some Hadamard-type inequalities, Journal of Inequalities in Pure and Applied Mathematics, vol. 5, no. 3, article 74, 2004.

[5] Dragomir, S. S., Pearce, C. E. M., Selected Topics on Hermite-Hadamard Inequalities and Applications, RGMIA Monographs, Victoria University, 2000.

[6] Dragomir, S. S., Agarwal, R.P., Two inequalities for differentiable mappings and applications to special means of real numbers and to trapezoidal formula, Appl. Math. Lett., 11(5) (1998), 91-95.

[7] Dragomir, S. S., On some new inequalities of Hermite-Hadamard type for $m-$ convex functions, Tamkang J. Math., 3(1) (2002).

[8] Dragomir, S. S., Fitzpatrik, S, .The Hadamard's inequality for $s$-convex functions in the second sense, Demonstratio Math. 32(4), (1999), 687-696.

[9] Gill, P. M. , Pearce, C. E. M., Pečarić, J., Hadamard's inequality for $r$-convex functions, Journal of Mathematical Analysis and Applications, vol. 215, no. 2, pp. 461--470, 1997.

[10] Hudzik, H., Maligranda, L., Some remarks on $s$-convex functions, Aequationes Math. 48 (1994), 100-111.

[11] Kirmaci, U.S., Bakula, M.K., Özdemir, M.E., Pečarić, J., Hadamard-tpye inequalities for $s$ convex functions, Appl. Math. and Comp., 193 (2007), 26-35.

[12] Toader, G, .Some generalizations of the convexity, Proceedings of The Colloquium On Approximation And Optimization, Univ. Cluj-Napoca, Cluj-Napoca,1985, 329-338.

[13] Özdemir, M. E., Avci, M., Set, E, .On some inequalities of Hermite-Hadamard type via $m$ convexity, Applied Mathematics Letters, vol. 23, no. 9, pp. 1065--1070, 2010.

[14] Pečarić, J.E., Proschan, F., Tong, Y.L., Convex Functions, Partial Orderings and Statistical Applications, Academic Press, Boston, 1992.

[15] Set, E., Özdemir, M. E. , Dragomir, S. S., On the Hermite-Hadamard inequality and other integral inequalities involving two functions, Journal of Inequalities and Applications, Article ID 148102, 9 pages, 2010.

[16] Set, E., Özdemir, M. E. , Dragomir, S. S., On Hadamard-Type inequalities involving several kinds of convexity, Journal of Inequalities and Applications, Article ID 286845, 12 pages, 2010.

[17] Mitrinović, D.S., Pečarić, J.E., Fink, A.M., Classical and New Inequalities in Analysis, Kluwer Academic Publishers, Dordrecht, 1993, p. 106.

[18] Anastassiou, G., Hooshmandasl, M.R., Ghasemi, A., Moftakharzadeh, F., Montogomery identities for fractional integrals and related fractional inequalities, J. Ineq. Pure and Appl. Math., 10(4) (2009), Art. 97.

[19] Belarbi, S., Dahmani, Z., On some new fractional integral inequalities, J. Ineq. Pure and Appl. Math., 10(3) (2009), Art. 86.

[20] Dahmani, Z., New inequalities in fractional integrals, International Journal of Nonlinear Scinece, 9(4) (2010), 493-497.

[21] Dahmani, Z., On Minkowski and Hermite-Hadamard integral inequalities via fractional integration, Ann. Funct. Anal. 1(1) (2010), 51-58.

[22] Dahmani, Z., Tabharit, L., Taf, S., Some fractional integral inequalities, Nonl. Sci. Lett. A, 1(2) (2010), 155-160.

[23] Dahmani, Z., Tabharit, L. , Taf, S., New generalizations of Gruss inequality usin RiemannLiouville fractional integrals, Bull. Math. Anal. Appl., 2(3) (2010), 93-99. 
[24] Gorenflo, R., Mainardi, F., Fractional calculus: integral and differential equations of fractional order, Springer Verlag, Wien (1997), 223-276.

[25] Miller, S., Ross, B, .An introduction to the Fractional Calculus and Fractional Differential Equations, John Wiley \& Sons, USA, 1993, p.2.

[26] Podlubni, I., Fractional Differential Equations, Academic Press, San Diego, 1999.

[27] Sarikaya, M.Z., Ogunmez, H. , On new inequalities via Riemann-Liouville fractional integration, Abstract and Applied Analysis, Volume 2012, Article ID 428983, 10 pages, doi:10.1155/2012/428983.

[28] Sarikaya, M.Z., Set, E., Yaldiz, H., Başak, N., Hermite-Hadamard's inequalities for fractional integrals and related fractional inequalities, Mathematical and Computer Modelling, 57 (2013), 2403--2407.

[29] Set, E., New inequalities of Ostrowski type for mapping whose derivatives are $s$-convex in the second sense via fractional integrals, Computers and Math. with Appl. 63 (2012), 1147-1154.

\section{Erhan Set,}

Department of Mathematics,

Faculty of Science and Arts,

Ordu University, Ordu, TURKEY,

e-mail: erhanset@yahoo.com

Mehmet Zeki Sarikaya,

Department of Mathematics,

Faculty of Science and Arts,

Düzce University, Düzce, TURKEY,

e-mail: sarikayamz@gmail.com

\section{Emin Özdemir}

Department of Mathematics

K. K. Education Faculty,

Ataturk University, 25640, Kampus, Erzurum, TURKEY,

e-mail: emos@atauni.edu.tr

Hüseyin Yıldırım

Department of Mathematics,

Faculty of Science and Arts,

Kahramanmaraş Sütcü Imam University, Kahramanmaraş-TURKEY,

e-mail: hyildir@ksu.edu.tr 